\title{
Vegetación asociada con helechales en el Parque Nacional Natural Serranía de Los Yariguíes, Colombia
}

\author{
Astrid Lorena Castro-Martínez ${ }^{1 *}$, Pablo Andrés Gil-Leguizamón ${ }^{1}$ \\ \& María Eugenia Morales-Puentes ${ }^{1}$ \\ 1. Sistemática Biológica, Herbario UPTC, Universidad Pedagógica y Tecnológica de Colombia, Avenida Central del \\ Norte39-115, Tunja, Boyacá, Colombia; astrid.castro01@uptc.edu.co, pablo.gil@uptc.edu.co, \\ maria.morales@uptc.edu.co \\ * Correspondencia
}

Recibido 31-I-2020. Corregido 21-III-2020. Aceptado 07-VIII-2020.

\begin{abstract}
Associated vegetation with bracken ferns in the Yariguíes National Natural Park, Colombia. Introduction: Alteration of the composition and structure of natural vegetation allows the invasion of species. This occurs with Pteridium esculentum subsp. arachnoideum x Pteridium esculentum subsp. campestre, which due to its growth by asexual reproduction and allelopathic effect, can quickly colonize disturbed habitat inhibiting natural succession, allowing herbaceous species to dominate and reducing tree species diversity. Objective: Evaluate the vegetation associated with the bracken P. esculentum subsp. arachnoideum $\mathrm{x}$ P. esculentum subsp. campestre distributed in the area called Golconda, in the Yariguíes National Park, Colombia. Methods: 15 plots of $25 \mathrm{~m}^{2}$ each were established; in each plot the vegetation composition and physiognomy were registered [growth forms, height $(\mathrm{m})$ as well as vegetation cover $\left(\mathrm{m}^{2}\right)$, diameter at breast height (DBH) for all trees and species abundance]; diversity probability indexes (Shannon and Simpson), percentage of species and abundance of growth forms, IVI for tree and shrub species, IVR for herbs, and the sampling effort were estimated. Results: 924 individuals belonging to 65 species were registered; they were distributed in 24 families; the richest taxa are: Melastomataceae, Asteraceae and Poaceae, which are considered frequent in disturbed areas. The areas dominated by ferns are botanically diverse, despite the dominance of species such as Ageratina cf. fastigiata, Tibouchina lindeniana, Clethra fagifolia, Coccocypselum lanceolatum and Clidemia ciliata; 30 woody and 21 herbaceous species were identified. Conclusions: Given that pioneer woody species and taxa from preserved areas of forest interiors subserve succession, this study contributes to a reevaluation of $P$. esculentum subsp. arachnoideum $\mathrm{x} P$. esculentum subsp. campestre as an inhibitor and cause of a decrease in diversity due to its dominance.
\end{abstract}

Key words: composition; structure; diversity; woody plants; ferns.

Castro-Martínez, A.L., Gil-Leguizamón, P.A., \& Morales-Puentes, M.E. (2020). Vegetación asociada con helechales en el Parque Nacional Natural Serranía de Los Yariguíes, Colombia. Revista de Biología Tropical, 68(4), 1107-1115.

Algunas comunidades de helechales son dominadas por especies de Pteridium y Dicranopteris, plantas con capacidad de establecerse posterior a la transformación de una formación vegetal original (Slocum, Aide, Zimmerman, \& Navarrro, 2000; Velázquez, 2001). Las alteraciones y pérdida de los bosques se debe a diferentes usos del suelo como agricultura, silvicultura, y ganadería, principales causas de transformaciones de la vegetación (Vitousek, Mooney, Lubchenco, \& Mellilo, 1997; Rodríguez, 2017; Tinjacá-Pérez, 2017); por lo anterior, estas especies colonizan rápidamente, por sus características heliófitas, acelerado crecimiento, y reproducción por esporas; además, son conocidas como invasoras, por la facultad 
inhibidora en los procesos de sucesión natural (Le Duc, Pakeman, Putwain, \& Marrs, 2000; Slocum et al., 2000; Hartig \& Beck, 2003).

Especies de Pteridium llamadas "helecho marranero o helecho macho" son consideradas invasoras e inhibidoras para el establecimiento en el proceso de sucesión, por ser de amplia distribución, activo crecimiento, colonización y capacidad competitiva (Alonso-Amelot, 1999; Velázquez, 2001; Ghorbani et al., 2003). Se desarrollan por estolones (tallo subterráneo) y producen sustancias alelopáticas que dificultan el establecimiento de plántulas de otras especies; razón por la cual, influyen directamente en la estructura y composición de una comunidad vegetal (Parks \& Werth, 1993; Gallegos et al., 2016). Es así como, Schwartsburd, Yanñez y Prado (2018) reorganizan los Pteridium de América del Sur, con criterios morfológicos y geográficos, lo cual, permite sinonimizar a P. arachnoideum del Parque Nacional Natural Serranía de Los Yariguíes (PNN SYA) bajo $P$. esculentum subsp. arachnoideum $\mathrm{x} P$. esculentum subsp. campestre, por caracteres como, venas abaxiales, indumento estrigoso y tricomas aciculares de $0.2-0.3 \mathrm{~mm}$ de longitud.

Según, Ghorbani et al. (2003), Gallegos, Hensen y Schleuning (2014) y Gallegos, Hensen, Saavedra y Schleuning (2015) en el trópico la diversidad de bosques en regeneración disminuye, debido a la dominancia de los helechos sobre las especies arbóreas y herbáceas; sin embargo, Gallegos et al. (2016) documentan que existe un predominio de especies herbáceas, mientras que la diversidad de especies arbóreas disminuye, ya que los helechos pueden permanecer en la sucesión temprana y limitar la sucesión tardía.

Recientemente, ha surgido el interés por conservar y preservar los ecosistemas, para amortiguar los efectos causados por las transformaciones en el suelo, la atmosfera y la biosfera (Vitousek et al., 1997; Lippok et al., 2013); profundizar en estos temas implica el conocimiento de la composición y la estructura de estas formaciones vegetales, soporte para implementar estrategias de restauración, y comprensión de ambientes perturbados (Fuentes, 2011).

PNN SYA es un área de reserva importante para Colombia, por su estado de conservación, diversidad, ecosistemas que posee, y zonas de vida (Holdridge, 1967; Donegan \& Huertas, 2005; Duarte, 2011; Rodríguez, 2017); es de interés para el PNN SYA los procesos de restauración ecológica, particularmente, en áreas dominadas por P. esculentum subsp. arachnoideum x P. esculentum subsp. campestre; por lo anterior, se presenta la composición y estructura florística de una comunidad de helechal en este Parque.

\section{MATERIALES Y MÉTODOS}

Área de estudio: El PNN SYA se ubica en la cordillera Oriental colombiana, departamento de Santander, y cubre siete municipios, incluido el Hato donde se realizó el estudio, en el área denominada Golconda. La extensión de Yariguíes es de 59063 ha, de las cuales, el $39 \%$ pertenecen a bosque, $35 \%$ a pastizales y $26 \%$ a cultivos, entre los 700-3 $400 \mathrm{~m}$, a los

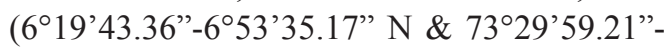
7321'29.01” W) (Donegan \& Huertas, 2005; Duarte, 2011).

Golconda tiene un rango de 2181-2 $188 \mathrm{~m}$,

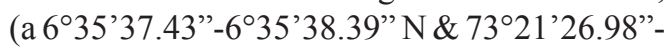
$\left.73^{\circ} 21^{\prime} 26.45^{\prime \prime} \mathrm{W}\right)$, con dominio de helechales, pastizales y relictos de bosque. También dominaban gramíneas exóticas para ganado, y cinco años atrás según pobladores, se evidenció la colonización del helecho (Caro, Gil, Alvarado, \& Morales, 2017).

Método: Se realizaron 15 parcelas de $25 \mathrm{~m}^{2}$ cada una (área total $=375 \mathrm{~m}^{2}$ ), en ellas se registraron datos de riqueza y abundancia por formas de crecimiento: árbol, arbusto, hierba, epifito, bejuco (Font Quer, 2000) y terrestre para clonales como helechos y plantas afines. La estructura horizontal, se determinó con datos de altura, cobertura, frecuencia y DAP para arbustos y árboles. Para cada parcela se tomaron datos de localidad, coordenadas, 
grados de inclinación y descripción del helechal, con el fin de definir el lugar de muestreo.

Los ejemplares se recolectaron en su mayoría con estructuras reproductivas. El material se identificó con claves especializadas (Gentry \& Vasquez, 1993; Steyermark, Berry, Holst, \& Yatskievych, 1995; Stevens, Ulloa, Pool, \& Montiel, 2001) y se corroboró la escritura científica con bases de datos como "Tropicos" (www.tropicos.org) y Jstor (www.jstor.org). Los exsicados se encuentran en el Herbario UPTC con duplicados en UIS, con numeración de Castro-Martínez A.L.

Análisis de datos: Para la composición y riqueza se identificó el total de familias, géneros y especies, las familias con mayor número de géneros y especies, $\mathrm{y}$ géneros con mayor riqueza. Se estimó la diversidad, con el índice de Shannon, dominancia de Simpson y la comparación entre parcelas con el índice de similitud de Jaccard, mediante el software PAST ver. $2.17 \mathrm{c}$.; se cuantificó la representatividad del muestreo con el promedio de los estimadores ACE, ICE, Chao 1, Chao 2, Jack 1, Jack 2, Bootstrap, para calcular las curvas de acumulación con uso de EstimateS ver. 9.1.0.

En cuanto a la estructura horizontal, se tomó en cuenta las formas de crecimiento dominantes según abundancia y riqueza; de igual forma, para identificar las especies arbóreas y arbustivas con mayor representatividad ecológica, se estimó el índice de valor de importancia (IVI según Rangel-Ch \& Velázquez, 1997), evaluado a partir de la abundancia, la frecuencia y la dominancia, y para las herbáceas, el índice de valor de importancia relativa (IVR, según; Álvarez-Lopeztello, Rivas-Manzano, Aguilera-Gómez, \& González-Ledesma, 2016).

\section{RESULTADOS}

Composición y diversidad: Se registraron 65 especies, distribuidas en 52 géneros y 24 familias; las familias más ricas son Melastomataceae (13 especies/6 géneros), Asteraceae (8/10), Poaceae (6/5), Cyperaceae (5/3),
Apocynaceae, Orchidaceae y Rubiaceae (3 especies cada una); familias como Campanulaceae, Ericaceae, Gentianaceae, Myrtaceae, Piperaceae con dos especies cada una, y las 12 restantes están representadas por un solo género y una especie (Apéndice digital 1). Las 12 familias más ricas corresponden al $77.4 \%$ de los géneros (41) y el $81.5 \%$ de especies (53). Los géneros más ricos son: Miconia (5 especies), Tibouchina (3), Rhynchospora (3), Clidemia (2), Baccharis (2), Piper (2), Andropogon (2) y Panicum (2); los 45 géneros restantes están representados por una especie.

El promedio de los estimadores de riqueza es del $91.31 \%$, siendo este un valor representativo para el área muestreada, por lo que, el valor de 3.178 para Shannon es representativo. Los valores de diversidad probable de Shannon entre parcelas no fueron contrastantes (de 2.21 a 2.89); es decir, estos datos determinaron baja diversidad en cada unidad de muestreo; lo anterior, debido a la alta dominancia (Simpson, entre 0.87 y 0.93). En la comunidad de helechal, las especies más abundantes son: Coccocypselum lanceolatum (147 individuos), Clethra fagifolia (92), Ageratina cf. fastigiata (73), Chromolaena tacotana (62) y Tibouchina lindeniana (54).

Similitud: Revela que es inferior al $54 \%$ entre la mayoría de las parcelas; solo cuatro tienen una similitud entre 70 y $72 \%$, siendo este último el valor máximo entre parcelas. La baja similitud indica alta heterogeneidad en las abundancias de las especies en cada parcela, lo que se asocia con recambio en composición.

Formas de crecimiento: Se registraron 924 individuos, en seis formas de crecimiento. De la abundancia total, 79 individuos estaban en etapa de plántula. Los arbustos son los más abundantes (363 individuos/39.3\%), seguido de hierbas $(342 / 37 \%)$ y terrestres $(112 / 12.2 \%)$. A nivel específico, las hierbas fueron las más representativas (21 sp./31.25 $\%)$, seguido de árboles (17/26.6\%), y arbustos (13/20.3\%); y aunque, los helechos presentan grandes coberturas $\left(6.25 \mathrm{~m}^{2}\right)$, su porcentaje 


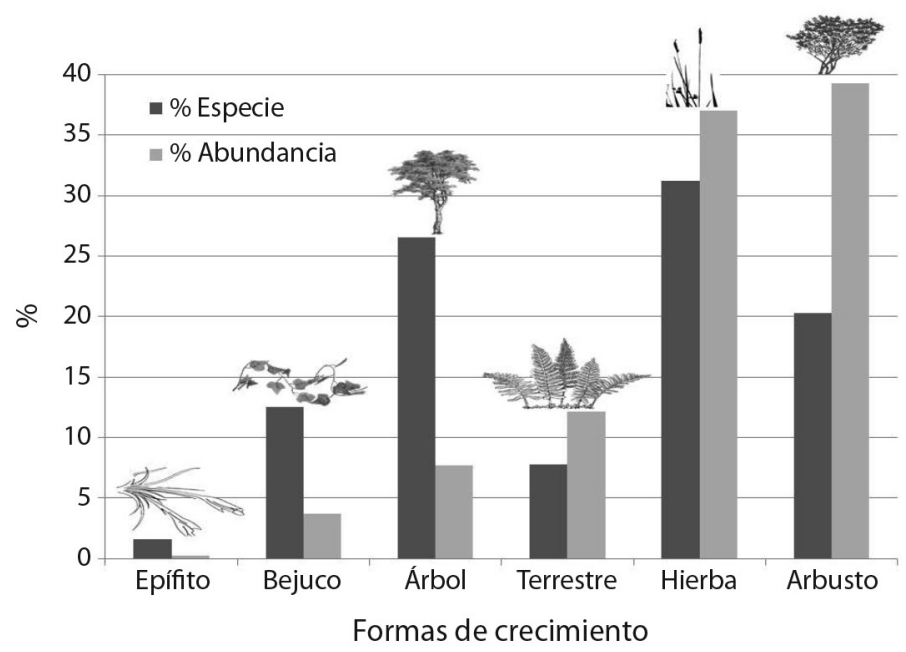

Fig. 1. Formas de crecimiento, abundancia y riqueza registrados en el área de estudio.

Fig. 1. Growth forms, abundance and richness recorded in the study area.

de especies es significativamente menor (5 sp./8.9\%) (Fig. 1).

Especies de importancia ecológica. Árboles y arbustos: Tibouchina lindeniana, Clethra fagifolia y Ageratina cf. fastigiata son las especies más representativas, aunque esta última, presenta el valor más alto de importancia por ser dominante $(74.42 \%)$.

Las especies dominantes son Tibouchina lindeniana (10.93\%) y T. lepidota (4.93\%), frecuentes y abundantes Ageratina cf. fastigiata (17.24\%/27.16\% respectivamente), Clethra fagifolia $(16.09 \% / 22.22 \%)$ у $T$. lindeniana $(12.64 \% / 19.75 \%)$; las especies restantes presentan valores de importancia inferiores al $20 \%$ (Fig. 2A).

Herbáceas: P. esculentum subsp. arachnoideum $\mathrm{x} P$. esculentum subsp. campestre $\mathrm{y}$ Urochloa decumbens son las más importantes debido a las coberturas relativas (43.6 y $32.8 \%$ respectivamente), seguido de Coccocypselum lanceolatum por abundancia (27.5\%) (Fig. 2B).

Los valores altos de coberturas los presentan, $P$. esculentum subsp. arachnoideum x P. esculentum subsp. campestre (43.6\%), $U$. decumbens (32.8\%) y Sticherus bifidus
(6.3\%); especies frecuentes son, P. esculentum subsp. arachnoideum x P. esculentum subsp. campestre, C. lanceolatum con el mismo valor (12.39\%), U. decumbens (11.57\%), Chromolaena tacotana $(9.91 \%)$ y Chaetolepis microphylla (9.09\%); mientras que las especies abundantes son: C. lanceolatum (27.5 \%), P. esculentum subsp. arachnoideum x $P$. esculentum subsp. campestre (16.61\%) y $U$. decumbens (13.46\%), y las restantes con valores inferiores al $10 \%$ (Fig. 2B).

\section{DISCUSIÓN}

Composición y diversidad: Los registros de 65 especies y 24 familias en el helechal ( $P$. esculentum subsp. arachnoideum x $P$. esculentum subsp. campestre) de Golconda (PNN SYA) (Apéndice digital 1), son similares a las cantidades identificadas por Ribeiro, Botelho, Leite Fontes, Garcia, \& Almeida (2012), en zonas con condiciones de dominancia de $P$. aquilinum ( $=P$. esculentum subsp. arachnoideum, Sierra de la Mantiqueira, Brasil), con 53 especies distribuidas en 21 familias; son comunes, Melastomataceae, Asteraceae, Myrsinaceae, Clethraceae y Myrtaceae, y géneros como Miconia, Tibouchina y Clethra; sin embargo, 

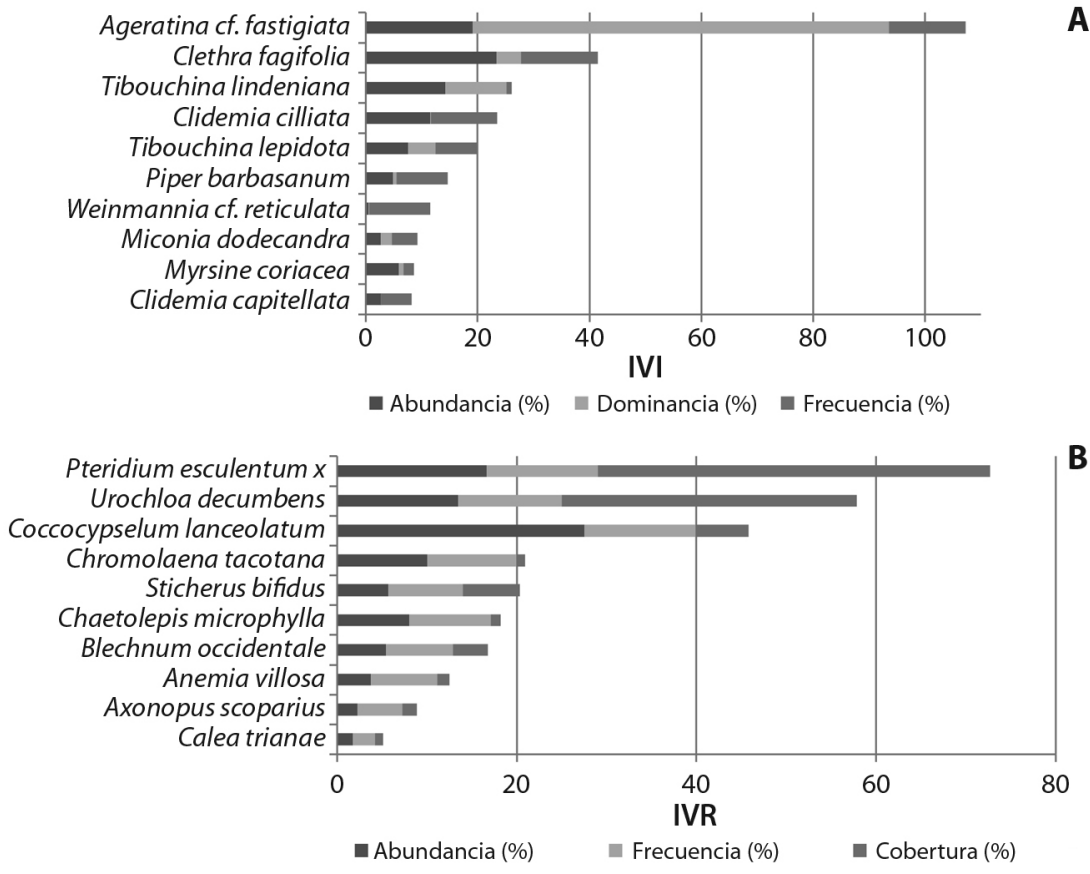

Fig. 2. Las 10 especies más importantes para el helechal ( $P$. esculentum subsp. arachnoideum x $P$. esculentum subsp. campestre) a partir del IVI e IVR. A. Árboles y arbustos B. Hierbas.

Fig. 2. The 10 most important species for bracken from IVI e IVR. P. esculentum subsp. arachnoideum $x$ P. esculentum subsp. campestre. A. Trees and shurbs B. Herbs.

Ribeiro et al. (2012), solo hizo muestreo de leñosas, por tal razón, este estudio presenta mayor riqueza, debido al estado de conservación del bosque andino e igualmente la composición difiere.

Melastomataceae, Poaceae y Asteraceae son representativas en riqueza, lo que concuerda con Hartig \& Beck (2003), Peters, Diertl, Gawlik, Rankl y Richter (2010) y Lippok et al. (2013), ya que, para algunas especies la dispersión anemócora les permite ser pioneras en la colonización de lugares perturbados. Según Ribeiro et al. (2012), Melastomataceae es rica y abundante, atribuido a la producción de semillas pequeñas y numerosas, característica que le permite sobrepasar barreras ecológicas y geográficas, y así, facilita su dispersión y establecimiento; a su vez, los helechos forman barreras físicas, que dificultan la colonización de especies leñosas, debido a la densidad de raíces en el suelo y cambios en la disponibilidad de nutrientes (Slocum et al., 2000); sin embargo, la presencia de estas familias determina procesos de recuperación natural en Golconda.

Especies como Weinmannia $\mathrm{cf}$. reticulata y Drimys granadensis propias de bosque fueron encontradas en helechal, lo que sugiere que el banco de semillas y/o la lluvia de semillas están aportando a la recuperación de esta formación; esto puede ser apoyado con el estudio de Tinjacá-Pérez (2017), donde se evaluó el banco de semillas de helechales, y concluye que estas formaciones vegetales no son limitantes en la regeneración natural, puesto que, el banco de semillas alberga gran diversidad y puede ser potencializado al controlar especies invasoras.

Similitud: La dominancia de Ageratina cf. fastigiata, T. lindeniana, C. fagifolia, C. lanceolatum y $C$. ciliata, coincide con los resultados obtenidos por diferentes autores, donde reportan dominancia para pocas especies (Slocum et al., 2000; Hartig \& Beck, 2003; Rodrigues Da Silva \& Silva Matos, 2006; 
Peters et al., 2010); lo anterior, atribuido a la competencia de Pteridium sp. y otros helechos, sobre especies leñosas que restringe el espacio para colonizar (Alonso-Amelot, 1999; Hartig \& Beck, 2003; Le Duc et al., 2000; Slocum et al., 2000); sin embargo, este estudio muestra que existe una diversidad representativa y es afectada por la heterogeneidad en las abundancias presentes en las parcelas según el análisis de Jaccard, y por la riqueza de especies, ya que, 20 de las familias encontradas presentan tres o menos especies.

Formas de crecimiento: Slocum et al. (2000), afirman que la diversidad de plantas arbóreas puede verse favorecida por el microclima generado por las frondas, y Hartig y Beck (2003), Peters et al. (2010) y Gallegos et al. (2016) concuerdan en que, las especies leñosas pioneras al establecerse, permiten la dispersión de semillas por aves.

Independiente del proceso de competencia, de barreras físicas y químicas que el helecho genera, o de la distancia entre las áreas de helechal y bosque (Parks \& Werth, 1993; AlonsoAmelot, 1999; Ghorbani et al., 2003; Gallegos et al., 2016), los resultados expuestos, permiten mostrar que existe dispersión y establecimiento de plántulas, evidenciado en la riqueza de leñosas encontradas (30 sp./65 sp. totales).

Se identificó alta abundancia y riqueza en herbáceas y leñosas, esta última con 513 individuos de 30 especies (Fig. 1), en contraste Slocum et al. (2000) afirman que, la vegetación leñosa asociada a helechales presenta menor abundancia y riqueza; lo que puede indicar que, los helechales de $P$. esculentum subsp. arachnoideum x P. esculentum subsp. campestre en Golconda no generan limitaciones para la sucesión temprana, al encontrarse especies pioneras, y tampoco en la sucesión tardía porque permite el establecimiento de especies leñosas; por el contrario, puede que actúe como una especie facilitadora en estos procesos.

Ghorbani, Le Duc, Mcallister, Pakeman y Marrs (2006) y Ribeiro et al. (2012), concluyeron que $P$. arachnoideum tiene una influencia negativa en la abundancia y riqueza de especies típicas de sucesión temprana, debido a las sustancias alelopáticas que produce y limitan el establecimiento de plántulas; sin embargo, las abundancias en este estudio son representativas en las formas de crecimiento arbustivo, herbáceo y arbóreo, lo cual puede indicar que, el banco de semillas presenta buena representación de diásporas y solo está latente, como lo indica también Tinjacá-Pérez (2017), o que las frondas, no obstruyen el arribo de especies por la lluvia de semillas; no obstante, la abundancia de plántulas es baja comparado con el número de individuos registrados.

Especies de importancia ecológica: Los índices IVI e IVR reflejan que las especies más importantes para el helechal son Ageratina cf. fastigiata (Asteraceae), P. esculentum subsp. arachnoideum x P. esculentum subsp. campestre (Dennstaedtiaceae), T. lindeniana (Melastomataceae), C. fagifolia (Clethraceae) y U. decumbens (Poaceae) (Figs. 2A, 2B), pertenecientes a las familias también reportadas por Rodrigues Da Silva \& Silva Matos (2006) y Ribeiro et al. (2012); puesto que, se caracterizan por tener dispersión anemócora, o esporas y semillas de tamaño relativamente pequeño que pueden colonizar con facilidad áreas que han pasado por disturbios.

Ageratina cf. fastigiata, tiene un valor de importancia alto, con DAP hasta de $10 \mathrm{~cm}$; de manera similar, las herbáceas como $P$. esculentum subsp. arachnoideum x $P$. esculentum subsp. campestre y $U$. decumbens tienen valores altos de importancia atribuidos a la cobertura, asociado a la reproducción asexual que permite la colonización de nuevas áreas; mientras que, las mayores abundancias son para C. lanceolatum común en zonas perturbadas y, C. fagifolia importante en los procesos de colonización por la dispersión de sus semillas pequeñas (Cardona \& Vargas, 2014).

La diversidad no disminuye a pesar de la dominancia de $P$. esculentum subsp. arachnoideum $\mathrm{x} P$. esculentum subsp. campestre, ya que se presenta una mayor riqueza respecto a otros estudios y, la abundancia es equitativa entre especies; de igual forma, se indica dominancia 
para especies leñosas como Ageratina cf. fastigiata, T. lindeniana, C. fagifolia y C. ciliata importantes en procesos de sucesión al ser claves en el arribo de especies; además se registran especies de interior de bosque que indican la recuperación de esta formación vegetal.

La riqueza de especies leñosas no se ve afectada por la presencia de P. esculentum subsp. arachnoideum x $P$. esculentum subsp. campestre, ya que éstas (pertenecientes a Melastomataceae y Asteraceae) pueden beneficiar la sucesión tardía; sin embargo, las abundancias de plántulas (79 respecto al total de la abundancia registrada) no son representativas; el helechal en Golconda posiblemente no impide el crecimiento y establecimiento de otras especies, sin embargo, dadas las características reproductivas y fisiológicas de $P$. esculentum subsp. arachnoideum $\mathrm{x} P$. esculentum subsp. campestre, posiblemente si restrinja el desarrollo rápido de los taxa, por lo cual, en términos de trayectorias ecológicas, direccionadas a la restauración de áreas disturbadas, estos helechales pudiesen ser considerados como un estado alternativo estable; lo anterior sugiere estudios respecto al efecto del helecho sobre el desarrollo de plántulas.

La importancia ecológica de Ageratina cf. fastigiata (por valor de IVI) está asociada con modificaciones en la composición y estructura del helechal y por ser facilitadora en la dispersión de semillas; de igual forma $P$. esculentum subsp. arachnoideum x $P$. esculentum subsp. campestre (por valores de IVR), genera un microambiente que permite la colonización de otras especies.

Declaración de ética: los autores declaran que todos están de acuerdo con esta publicación y que han hecho aportes que justifican su autoría; que no hay conflicto de interés de ningún tipo; y que han cumplido con todos los requisitos y procedimientos éticos y legales pertinentes. Todas las fuentes de financiamiento se detallan plena y claramente en la sección de agradecimientos. El respectivo documento legal firmado se encuentra en los archivos de la revista.

\section{AGRADECIMIENTOS}

Al convenio 5211740 Uptc-Ecopetrol (proy. Restauración ecológica de 16,18 ha en PNN SYA). A la Territorial Nororiental de PNN. A la Universidad Pedagógica y Tecnológica de Colombia, a SisBio y Herbario UPTC. A la comunidad de Hoya Negra (Hato-Santander), y a J. Afanador, F. Corzo e I. Cala.

\section{RESUMEN}

Introducción: La alteración en la composición y estructura de la vegetación da paso a la introducción de especies consideradas invasoras, caso de Pteridium arachnoideum, que, por sus características de crecimiento por reproducción asexual y efecto alelopático, puede colonizar rápidamente; tal capacidad de invasión, inhibe los procesos de sucesión natural, permite el dominio de especies herbáceas y disminuye la diversidad de especies arbóreas. Objetivo: Evaluar la vegetación asociada a los helechales (P. esculentum subsp. arachnoideum $\mathrm{x}$ P. esculentum subsp. campestre) distribuidos en la zona denominada Golconda, ubicada en el Parque Nacional Natural Serranía de Los Yariguíes. Métodos: Se realizaron 15 parcelas de $25 \mathrm{~m}^{2}$ cada una; en ellas, se tomaron datos de composición y fisonomía de la vegetación [formas de crecimiento, altura (m), cobertura $\left(\mathrm{m}^{2}\right)$, diámetro a la altura del pecho (DAP) para todos los árboles y abundancia de especies]; se estimaron índices de probabilidad de diversidad (Shannon y Simpson), el porcentaje de especies y abundancia por formas de crecimiento, IVI para especies arbóreas y arbustivas, IVR para herbáceas y el esfuerzo de muestreo. Resultados: Se registraron 924 individuos pertenecientes a 65 especies, distribuidas en 24 familias; las taxa más ricas son: Melastomataceae, Asteraceae y Poaceae, consideradas frecuentes en áreas perturbadas. Las áreas dominadas por helechales son diversas, a pesar de la dominancia de especies como: Ageratina cf. fastigiata, Tibouchina lindeniana, Clethra fagifolia, Coccocypselum lanceolatum y Clidemia ciliata; se identificaron 30 especies leñosas y 21 herbáceas. Conclusiones: Con especies leñosas pioneras que favorecen la sucesión, así como, taxones provenientes de áreas conservadas de interior de bosque, este estudio contribuye en la reevaluación del atributo de $P$. esculentum subsp. arachnoideum $\mathrm{x} P$. esculentum subsp. campestre como inhibidora y causante de la disminución de la diversidad por su dominancia.

Palabras clave: composición; estructura; diversidad; plantas leñosas; helechos. 


\section{REFERENCIAS}

Alonso-Amelot, M.E. (1999). Helecho macho, salud animal y salud humana. Revista Facultad de Agronomía, $16,528-541$.

Álvarez-Lopeztello, J., Rivas-Manzano, I.V., AguileraGómez, L.I., \& González-Ledesma, M. (2016). Diversidad y estructura de un pastizal en El Cerrillo, Piedras Blancas, Estado de México, México. Revista Mexicana de Biodiversidad, 87(3), 980-989.

Cardona, A., \& Vargas, O. (2014). El banco de semillas germinable de especies leñosas en dos Bosques Subandinos y su importancia para la restauración ecológica (Reserva Biológica Cachalú-Santander Colombia). Colombia Forestal, 8(17), 60-74.

Caro, D.P., Gil, P.A., Alvarado, V.M., \& Morales, M.E. (2017). Avances en estudios de la flora vascular, flanco oriental de la Serranía de Los Yariguíes (Hato) Santander-Colombia. Ciencia en Desarrollo, 1E, 656-657.

Donegan, T., \& Huertas, B. (2005). Threatened species of Serranía de los Yariguies: Final Report. Bogotá, Colombia: Fundación ProAves.

Duarte, I. (2011). Diagnóstico de los aspectos físicobióticos del Parque Nacional Natural Serranía de los Yariguies, Santander-Colombia. San Vicente de Chucurí, Colombia.

Font Quer, P. (2000). Diccionario de botánica. Barcelona, España: Editorial Península.

Fuentes, B.A. (2011). Estrategia de restauración ecológica participativa del sistema de Parques Nacionales Naturales de Colombia. En O. Vargas \& S.P. Reyes (Eds.), La restauración ecológica en la práctica: Memorias I Congreso Colombiano de Restauración Ecológica \& II Simposio Nacional de Experiencias en Restauración Ecológica (p. 633). Bogotá D.C., Colombia: Universidad Nacional de Colombia.

Gallegos, S.C., Beck, S.G., Hensen, I., Saavedra, F., Lippok, D., \& Schleuning, M. (2016). Factors limiting montane forest regeneration in bracken-dominated habitats in the tropics. Forest Ecology and Management, 381, 168-176.

Gallegos, S.C., Hensen, I., Saavedra, F., \& Schleuning, M. (2015). Bracken fern facilitates tree seedling recruitment in tropical fire-degraded habitats. Forest Ecology and Management, 337, 135-143.

Gallegos, S.C., Hensen, I., \& Schleuning, M. (2014). Secondary dispersal by ants promotes forest regeneration after deforestation. Journal of Ecology, $102(3), 659-666$

Gentry, A.H., \& Vasquez, R. (1993). A field guide to the families and genera of woody plants of northwest South America (Colombia, Ecuador, Peru): with supplementary notes on herbaceous taxa. Chicago, USA: University of Chicago Press.

Ghorbani, J., Le Duc, M.G., Mcallister, H.A., Pakeman, R.J., \& Marrs, R.H. (2006). Effects of the litter layer of Pteridium aquilinum on seed banks under experimental restoration. Applied Vegetation Science, 9(1), 127-136.

Ghorbani, J., Das, P.M., Das, A.B., Hughes, J.M., McAllister, H.A., Pallai, S.K., ... Le Duc, M.G. (2003). Effects of restoration treatments on the diaspore bank under dense Pteridium stands in the UK. Applied Vegetation Science, 6(2), 189-198.

Hartig, K., \& Beck, E. (2003). The bracken fern (Pteridium arachnoideum (Kaulf.) Maxon) dilemma in the Andes of Southern Ecuador. Ecotropica, 9, 3-13.

Holdridge, L.R. (1967). Life zone ecology. San José, Costa Rica: Tropical Science Center.

Le Duc, M.G., Pakeman, R.J., Putwain, P.D., \& Marrs, R.H. (2000). The variable responses of bracken fronds to control treatments in Great Britain. Annals of Botany, 85(Suppl. B), 17-29.

Lippok, D., Beck, S.G., Renison, D., Gallegos, S.C., Saavedra, F.V., Hensen, I., \& Schleuning, M. (2013). Forest recovery of areas deforested by fire increases with elevation in the tropical Andes. Forest Ecology and Management, 295, 69-76.

Parks, J.C., \& Werth, C.R. (1993). A study of spatial features of clones in a population of bracken fern, Pteridium aquilinum (Dennstaedtiaceae). American Journal of Botany, 80(5), 537-544.

Peters, T., Diertl, K.H., Gawlik, J., Rankl, M., \& Richter, M. (2010). Vascular plant diversity in natural and anthropogenic ecosystems in the Andes of southern Ecuador. Mountain Research and Development, 30(4), 344-352.

Rangel-Ch, O., \& Velázquez, A. (1997). Métodos de estudio de la vegetación. En O. Rangel, P. Lowly, \& M. Aguilar (Eds.), Colombia Diversidad Biótica II: Tipos de vegetación en Colombia (pp. 59-87). Bogotá, Colombia: Universidad Nacional de Colombia.

Ribeiro, S.C., Botelho, S.A., Leite Fontes, M.A., Garcia, P.O., \& Almeida, H. (2012). Regeneração natural em áreas desmatadas e dominadas por Pteridium aquilinum (L.) Kuhn. na Serra da Mantiqueira. Cerne, 19(1), 65-76.

Rodrigues Da Silva, Ú.D.S., \& Silva Matos, D.M.D. (2006). The invasion of Pteridium aquilinum and the impoverishment of the seed bank in fire prone areas of Brazilian Atlantic Forest. Biodiversity and Conservation, 15(9), 3035-3043.

Rodríguez, W.D. (2017). Familia Dennstaeditaceae Pteridium aquilinum (L.) Kuhn. En D. Cárdenas-L, M.P. 
Baptiste, \& N. Castaño (Eds.), Plantas exóticas con alto potencial de invasión en Colombia (pp. 102106). Bogotá, Colombia: Instituto de Investigación de Recursos Biológicos Alexander von Humboldt.

Schwartsburd, P.B., Yañez, A. \& Prado, J. (2018). Formal recognition of six subordinate taxa within the South American bracken fern, Pteridium esculentum ( $P$. esculentum subsp. arachnoideum s.l.-Dennstaedtiaceae), based on morphology and geography. Phytota$x a, 333(1), 022-040$.

Slocum, M., Aide, M., Zimmerman, J.K., \& Navarro, L. (2000). La vegetación leñosa en helechales y bosque de ribera en la reserva científica Ébano Verde, República Dominicana. Moscosoa, 11, 38-56.

Stevens, W.D., Ulloa, C., Pool, A. \& Montiel, O.M. (2001). Flora de Nicaragua (Monographs in Systematic
Botany from the Missouri Botanical Garden). Saint Luis, USA: Missouri Botanical Garden Press.

Steyermark, J.A., Berry, P.E., Holst, B.K., \& Yatskievych, K. (1995). Flora of the Venezuelan Guayana. Saint Louis, USA: Missouri Botanical Garden.

Tinjacá-Pérez, L. (2017). Potencial de regeneración natural del bosque húmedo montano en áreas de pastizal $y$ helechal en el PNN Serrania de los yariguies, Santander-Colombia (Trabajo de pregrado). Pontificia Universidad Javeriana, Bogotá D.C., Colombia.

Velázquez, E. (2001). Pteridium un género de helechos muy particular. Ciencias, 64, 21.

Vitousek, P., Mooney, H.A., Lubchenco, J., \& Mellilo, J.M. (1997). Human domination of Earth's ecosystems. Science, 227, 494-499.

See Digital Appendix at: / Ver Apéndice digital en:

revistas.ucr.ac.cr 